\title{
Wetware, Hardware, or Software Incapacitation: Observational Methods to Determine When Autonomy Should Assume Control
}

\author{
Anna Trujillo ${ }^{1}$ and Irene Gregory ${ }^{2}$ \\ NASA Langley Research Center, Hampton, VA, 23681
}

\begin{abstract}
Control-theoretic modeling of human operator's dynamic behavior in manual control tasks has a long, rich history. There has been significant work on techniques used to identify the pilot model of a given structure. This research attempts to go beyond pilot identification based on experimental data to develop a predictor of pilot behavior. Two methods for predicting pilot stick input during changing aircraft dynamics and deducing changes in pilot behavior are presented This approach may also have the capability to detect a change in a subject due to workload, engagement, etc., or the effects of changes in vehicle dynamics on the pilot. With this ability to detect changes in piloting behavior, the possibility now exists to mediate human adverse behaviors, hardware failures, and software anomalies with autonomy that may ameliorate these undesirable effects. However, appropriate timing of when autonomy should assume control is dependent on criticality of actions to safety, sensitivity of methods to accurately detect these adverse changes, and effects of changes in levels of automation of the system as a whole.
\end{abstract}

\section{Nomenclature}

ACE-PE = Adaptive Controller Effects on Pilots Experiment

$\mathrm{GDE}=$ Gradient Descent Estimator

LSEEF $=$ Least Squares Estimator with Exponential Forgetting

LTE $\quad=$ Longitudinal Tracking Experiment

$\mathrm{SE} \quad=$ Standard Error of the Mean

$\Gamma_{\Theta} \quad=$ Estimation Gain for the Gradient Descent Estimator

\section{Introduction}

Significant research has been conducted to model or identify the pilot, as a way to quantify handling qualities or $\checkmark$ to better understand the behavior of a human pilot in controlling a vehicle ${ }^{1-7}$. The classical McRuer crossover model of compensatory manual control states that the human operator will adapt to vehicle dynamics by providing lead equalization, with an associated computational penalty, such that the combined operator-vehicle transfer function is proportional to an integrator at the crossover frequency ${ }^{7}$. Another approach to understanding the human-asthe-controller behavior was the development of the optimal control model by Kleinman, Baron, and Levinson based on the assumption that a well-trained and motivated human controller behaves optimally in some sense, adjusting the pilot's compensation for a given vehicle and task, subject to human limitations ${ }^{8}$.

In the last two decades, there has been a renewed interest in modeling the human operator. If the human operator can be adequately represented, this will shorten the development cycle, especially in control law development. Another benefit of human operator modeling would be to safely test in various regimes, such as stall and recovery, and to investigate accidents. In fact, an accident investigation led Hess to further develop a human operator model ${ }^{9}$. The Hess modeling in the frequency domain, a structural model ${ }^{10}$, and Schmidt working in the time domain, a modified optimal control pilot model ${ }^{2,11}$, attempted to take into consideration appropriate relevant feedback that influenced a pilot's behavior such as proprioceptive cues, and visual and vestibular feedback. While these models proved useful, their application to realistic simulation tasks involved a great deal of complexity. In an attempt to simplify the model

\footnotetext{
${ }^{1}$ Senior Research Engineer, Crew Systems and Aviation Operations Branch, MS 152, AIAA Member.

${ }^{2}$ Senior Research Engineer, Langley Intelligent Flight Systems Lead, MS 308, AIAA Associate Fellow.
}

1

American Institute of Aeronautics and Astronautics 
while still capturing the essential pilot behavioral characteristics, Hess proposed a simplified model that has been recently applied to systems with time-varying dynamics ${ }^{12,13}$.

In addition, to several relevant model structures to describe the pilot as a controller behavior, there has also been significant work on techniques used to identify the pilot model of a given structure ${ }^{14-20}$. These included various applications of parameter identification ranging from wavelets-based approach to frequency-based system identification techniques.

Various models have also been developed using a physiological basis, such as the man-machine integration design and analysis system (MIDAS) $)^{21-23}$. These "human performance modelling process have attempted to integrate operator characteristics (cognitive, attentional, and physical) with environmental characteristics to more accurately represent human-system operations with new, augmented technologies" ${ }^{\prime 2}$. They typically involve higher-level decision making models and processes rather than more immediate, direct control actions.

This research is investigating new analytical methods to model the pilot's changing behavior over short time periods in response to changing aircraft dynamics. This research is attempting to go beyond pilot identification based on collected experimental data and to develop a predictor of short-term pilot control behavior in real time. The long term goal of this research is to describe a model that goes beyond identifying pilot behavior changes due to vehicle dynamic changes but captures changes in pilot's behavior due to a myriad of factors, such as pilot incapacitation. This may enable identification of potential reasons for this behavior change; hence, informing the decision process for changing function allocation between pilot and automation.

Availability of such an analytical model would have impact in a number of different areas. Obviously, it would contribute to potential requirements for adaptive control law design helping to ameliorate hardware failures. In more pilot-centric arenas, this analytical model may affect the design of decision aids for the pilot by considering the pilot's control response or method of piloting the aircraft. It may also help determine the appropriate function allocation between the pilot and the automation especially during changes in effective dynamics. The analytical models, if similarly applied to autopilots, may also be able to detect software glitches with the automation. Once the system is able to detect these failures, appropriate timing of when autonomy should assume control is dependent on criticality of actions to safety, sensitivity of methods to accurately detect these adverse changes, and effects of changes in levels of automation of the system as a whole.

\section{Analytical Model Development}

Data from two separate experiments, briefly described below in Sections IIA and IIB, were used to estimate vehicle pitch attitude from pilot longitudinal stick input. The estimation methods, described in Section IIC below, were a gradient descent estimator and a least squares estimator with exponential forgetting.

\section{A. Longitudinal Tracking Experiment}

The longitudinal tracking experiment (LTE) was conducted to quantify the effects of changing dynamics on a subject's ability to track a signal in order to eventually model a pilot adapting to changing aircraft dynamics ${ }^{24}$. Each run incorporated a different set of second-order aircraft dynamics representing short period transfer function pitch attitude response with a potential system time delay: damping ratio, frequency, gain, zero location, and time delay. The aircraft short-period attitude transfer function was:

$$
\frac{\theta}{\delta}(s)=\frac{K\left(s+L_{\alpha}\right)}{s\left[(s / \omega)^{2}+2 s(s / \omega)+1\right]} e^{-\tau s}
$$

where $\theta$ is pitch attitude and $\delta$ is pilot stick. The damping ratio $(\zeta)$ was $0.4,0.7$, or 1 . The short period frequency $(\omega)$ was $0.5 \mathrm{~Hz}, 1 \mathrm{~Hz}$, or $1.5 \mathrm{~Hz}$. Gain $(K)$ was 1,2 , or 3 . Zero location $\left(L_{\alpha}\right)$ was $0.5,1$, or 1.5 . Lastly, time delay $\tau$ was 0 $\mathrm{ms}, 75 \mathrm{~ms}$, or $150 \mathrm{~ms}$. For a detailed description of this experiment, see Trujillo and Gregory ${ }^{24}$.

\section{B. Adaptive Controller Effects on Pilots Experiment}

The adaptive controller effect on pilots experiment (ACE-PE) was conducted to categorize the interactions between the pilot and an adaptive controller during change in aircraft dynamics represented by control surface failures ${ }^{25-27}$. One of the objectives of this experiment was to determine how the adaptation time of the controller affects pilots. This was accomplished by considering whether an adaptive controller helps pilots during control sur- 
face failures (by measuring tracking error) and how this controller affects pilots before, during, and after the control surface failures. The control surface failures were either stuck surfaces or slowly moving surfaces. A human-in-theloop experiment looked at the effects of these control surface failures on pilot performance during cruise phase while initiating a climb, descent, or a heading change maneuver. These maneuvers were indicated on the primary flight display (PFD) via the flight director and on the engine indication display's (EID) horizontal and vertical navigation map displays. Each subject experienced four adaptation times: zero seconds, three seconds, seven seconds, and no adaptation (Never). These times indicated how long it took the adaptive controller-aircraft system to settle to a new set of dynamics and specific values are based on the response speed of the nominal aircraft dynamics. Zero seconds indicated the fastest possible adaptation time, essentially the processor speed. For a detailed description of this experiment, see Trujillo and Gregory ${ }^{25}$, Trujillo and Gregory ${ }^{26}$, and Trujillo, et al. ${ }^{27}$ ENREF 27.

\section{Estimation Methods}

In both LTE and ACE-PE, the subjects' longitudinal stick inputs and longitudinal flight director commands were recorded. With this information, a pilot input-output model was built using a gradient descent estimator (GDE) and a least squares estimator with exponential forgetting (LSEEF) ${ }^{28}$ techniques. The online implementation of the GDE is given by

$$
\dot{\hat{\theta}}(t)=-\Gamma_{\Theta} \Phi(x(t))(\underbrace{\Phi(x(t))^{T} \hat{\theta}(t)}_{\hat{y}(t)}-y(t))
$$

where $y(t)$ and $x(t)$ are system output (longitudinal stick) and input signal (pitch command) respectively, $\Phi(x(t))$ is the regressor/basis function, $\Gamma_{\Theta}$ is the estimation gain, $\hat{\theta}$ is the estimated parameter, and $\hat{y}(t)=\Phi(x(t))^{T} \theta(t)$ represents the predicted/estimated output of the model at time $t$. Note longitudinal stick deflection was normalized to \pm 1 range. Similarly, the online representation of the LSEEF is

$$
\left\{\begin{array}{l}
\dot{\hat{\theta}}(t)=-\Gamma_{\Theta}(t) \Phi(x(t))\left(\Phi(x(t))^{T} \hat{\theta}(t)-y(t)\right), \quad \hat{\theta}(0)=\hat{\theta}_{0} \\
\dot{\Gamma}_{\Theta}(t)=\lambda(t) \Gamma_{\Theta}(t)-\Gamma_{\Theta}(t) \Phi(x(t)) \Phi(x(t))^{T} \Gamma_{\Theta}(t), \quad \Gamma_{\Theta}(0)=\Gamma_{\Theta 0}
\end{array} .\right.
$$

The variable $\lambda(t)$ is part of exponential forgetting factor which enables LSEEF to track time-varying parameters, albeit slowly varying. The rest of the variables are the same as described above for GDE For GDE, the estimation gain, $\Gamma_{\Theta}$, was recursively calculated for each subject's run until the estimated longitudinal stick input matched the actual longitudinal stick input as close as possible. The estimation gain, $\Gamma_{\Theta}$, for LSEEF, was also recursively calculated for each subject's run until the estimated longitudinal stick input matched the actual stick input as close as possible.

\section{Results}

The below data analyses were done with IBM SPSS ${ }^{\circledR}$ Statistics version $22^{29}$. An analysis of variance (ANOVA) was used for statistical testing. Significance for all tests was set at $\mathrm{p} \leq 0.05$. Lastly, any error bars present in a graph indicate one standard error of the mean (SE).

\section{A. Estimation Method Accuracy}

The two estimation methods were compared by computing the error between the actual longitudinal stick input and the estimated longitudinal stick input from each of the two methods - GDE and LSEEF using the LTE data (see Section IIA above).

When comparing the error between the predicted longitudinal stick input and the actual longitudinal stick input, the GDE method had the least error $\left(F_{2,612}=157.28 ; \mathrm{p} \leq 0.001\right)$ (Table 1$)$. An example com-

Table 1. Estimation Method Accuracy

\begin{tabular}{|c|c|}
\hline $\begin{array}{c}\text { Estimation } \\
\text { Method }\end{array}$ & $\begin{array}{c}\text { Mean Error } \pm \text { 1 SE } \\
\text { (Predicted Longitudinal Stick Input - } \\
\text { Actual Longitudinal Stick Input) }\end{array}$ \\
\hline GDE & $0.046 \pm 0.00387$ \\
\hline LSEEF & $0.112 \pm 0.00384$ \\
\hline
\end{tabular}


paring the stick inputs of the two methods to the actual pitch output is shown in Figure 1.
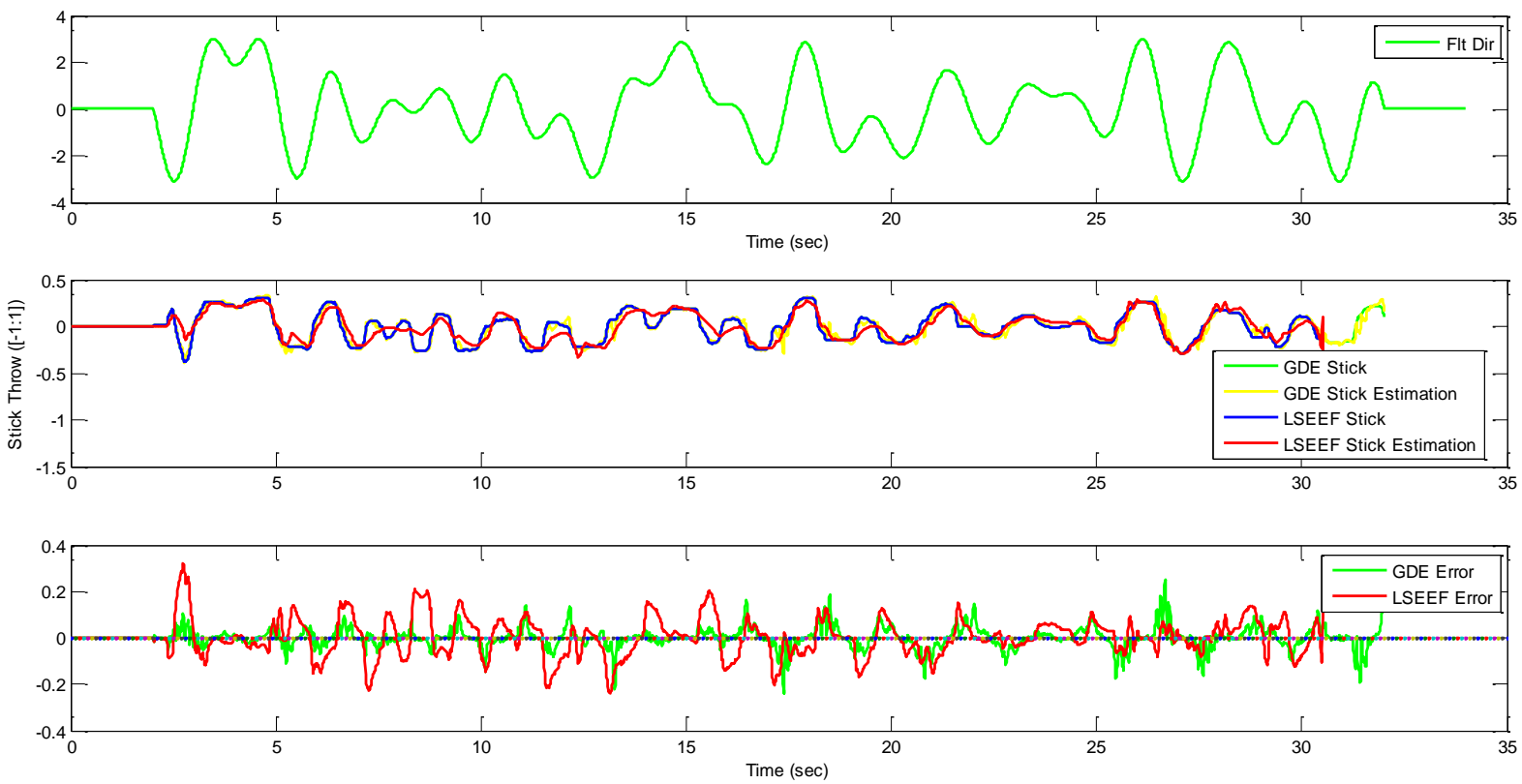

Figure 1. Top Graph: Longitudinal Flight Director Command Input Middle Graph: Actual and Predicted Longitudinal Stick Input for GDE and LSEEF Bottom Graph: Predicted-Actual Longitudinal Stick Input for GDE and LSEEF

Furthermore, the GDE adaptation parameter, $\Gamma_{\Theta}$, was affected by the subject $\left(F_{3,198}=23.78 ; \mathrm{p} \leq 0.001\right)$ (Figure 2$)$, short-period damping $\zeta\left(F_{1,198}=5.78 ; \mathrm{p}=0.017\right)$ (Figure 3 ), and was almost statistically dependent on system time delay $\tau\left(F_{1,198}=3.13 ; \mathrm{p}=0.079\right)$ (Figure 3$)$. Hence, for a set of data with constant dynamics for the entirety of a run, the adaptation gain is dependent on the subject and on a particular set of dynamics. The same model was then applied to a set of dynamics that varied during a run. The GDE method to predict the longitudinal stick input was used on another set of data from ACE-PE that involved an adaptive controller responding to surface failures where adaptation time was preset to four specified values before the controller-aircraft system settled on a new set of dynamics.

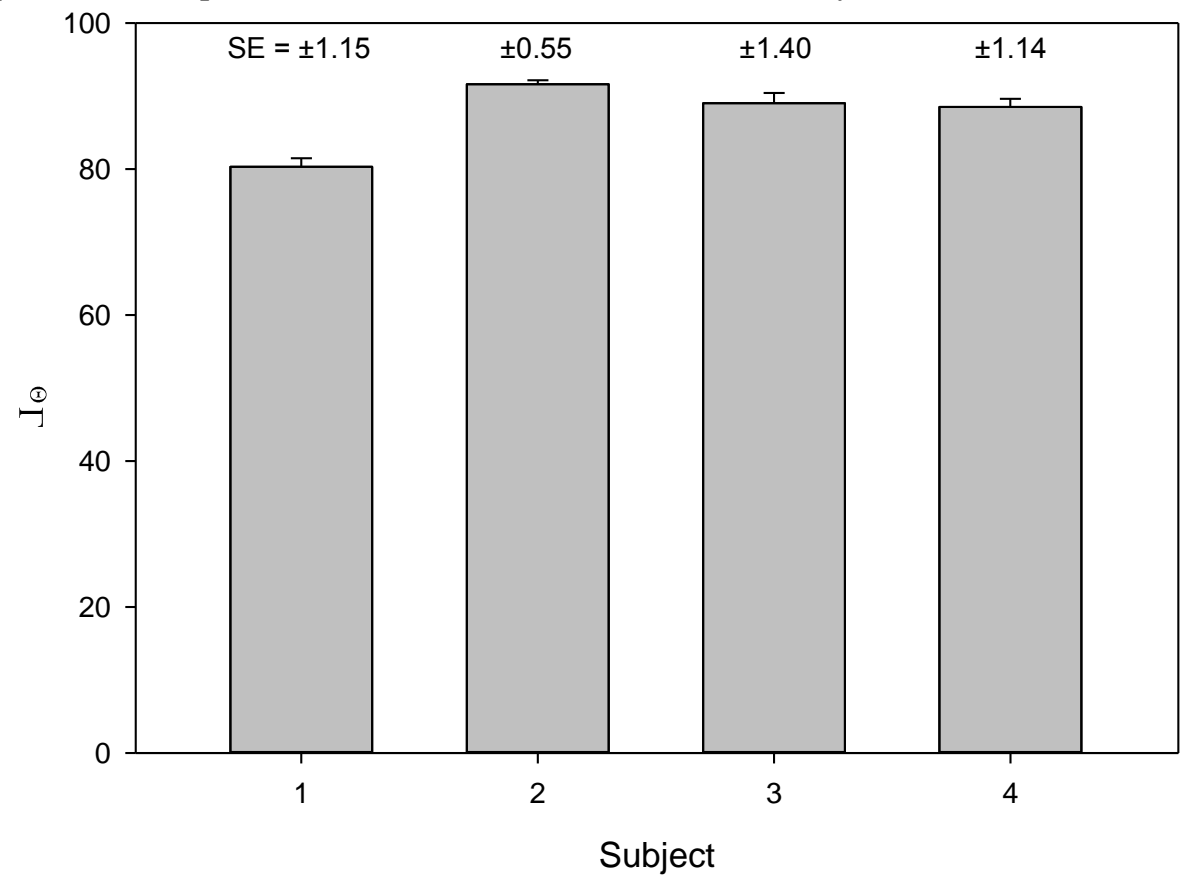

Figure 2. $\Gamma_{\Theta}$ by Subject

American Institute of Aeronautics and Astronautics 


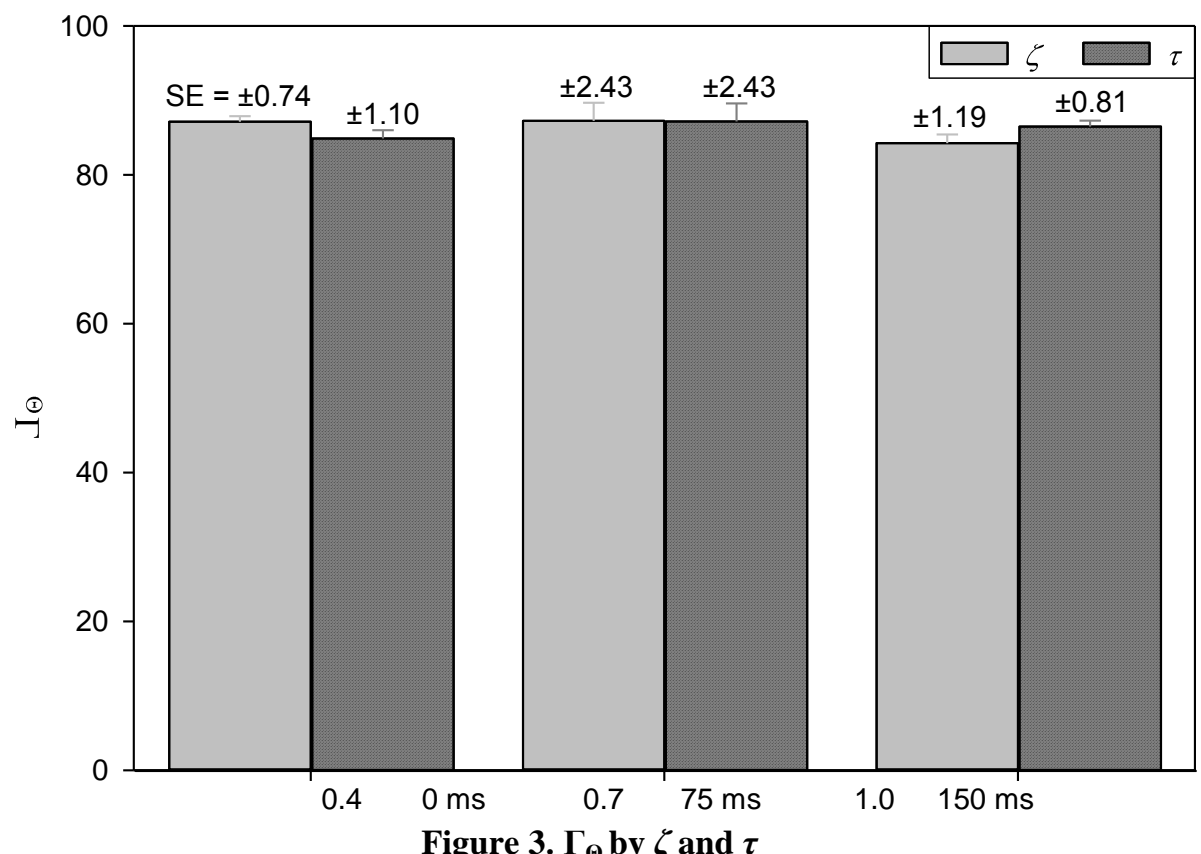

\section{B. Detecting Changes in Pilot Longitudinal Output in the ACE-PE Data}

The GDE method was used on the ACE-PE data (see Section IIB above). As with the LTE data, the input data was the longitudinal flight director command and the estimated output was the longitudinal stick input. The GDE parameter $\Gamma_{\Theta}$ was calculated for each subject's runs.

The GDE parameter $\Gamma_{\Theta}$ is dependent on who is piloting $\left(F_{16,2773}=39.98 ; \mathrm{p} \leq 0.001\right)$ although there is overlap among the six groups (Figure 4) defined by the Tukey Honestly Significant Difference post-hoc test. When looking at the data, these six overlapping groups appear to consist of three primary groups - subjects with a low $\Gamma_{\Theta}$ value, subjects with a medium $\Gamma_{\Theta}$ value, and subjects with a high $\Gamma_{\Theta}$ value (Figure 4). This suggests that there may be a finite set of methods pilots use to control a vehicle such as continuously minimizing error or waiting until the error has grown by a certain amount before quickly attempting to zero out the error.

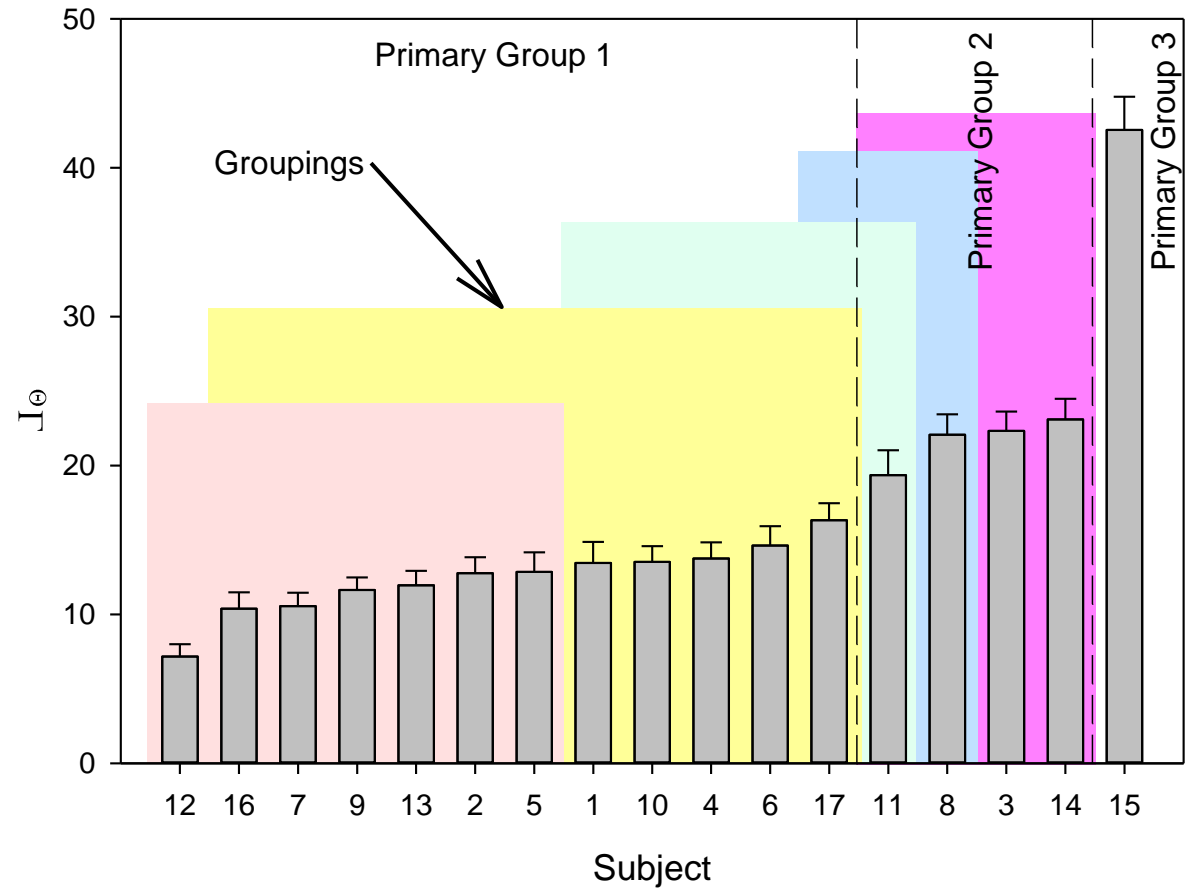

Figure 4. $\Gamma_{\Theta}$ by Subject with Groupings

American Institute of Aeronautics and Astronautics 
Each data run is comprised of three segments: before failure, during failure and after failure. The GDE estimation parameter $\Gamma_{\Theta}$ did change dependent on the segment and subject $\left(F_{32,2773}=3.66 ; \mathrm{p} \leq 0.001\right)$ (Figure 5). In general, $\Gamma_{\Theta}$ increased during the failure segment and then started to decrease after the failure segment $\left(F_{2,2773}=48.72\right.$; $\mathrm{p} \leq 0.001)$ as can be seen in Figure 5 and Figure 6 . Eventually, $\Gamma_{\Theta}$ may return to its value before the failure occurred (Figure 6 "Difference in $\Gamma_{\Theta}$ ") $\left(F_{1,1663}=80.32 ; \mathrm{p} \leq 0.001\right)$ provided post-failure controller-aircraft dynamics return to nominal aircraft . Oddly enough, though, the difference in $\Gamma_{\Theta}$ was not the highest for the case when the adaptive controller never adapted $\left(F_{3,1759}=66.72 ; \mathrm{p} \leq 0.001\right)$ (Figure 7$)$. Further data analysis is required to explain why essentially instantaneous adaptation indicated pilot behavior change, which has been confirmed by other measures ${ }^{24}$. Despite this anomaly, the results indicate that the amount of time the off-nominal condition is in effect will influence the magnitude of $\Gamma_{\Theta}$.

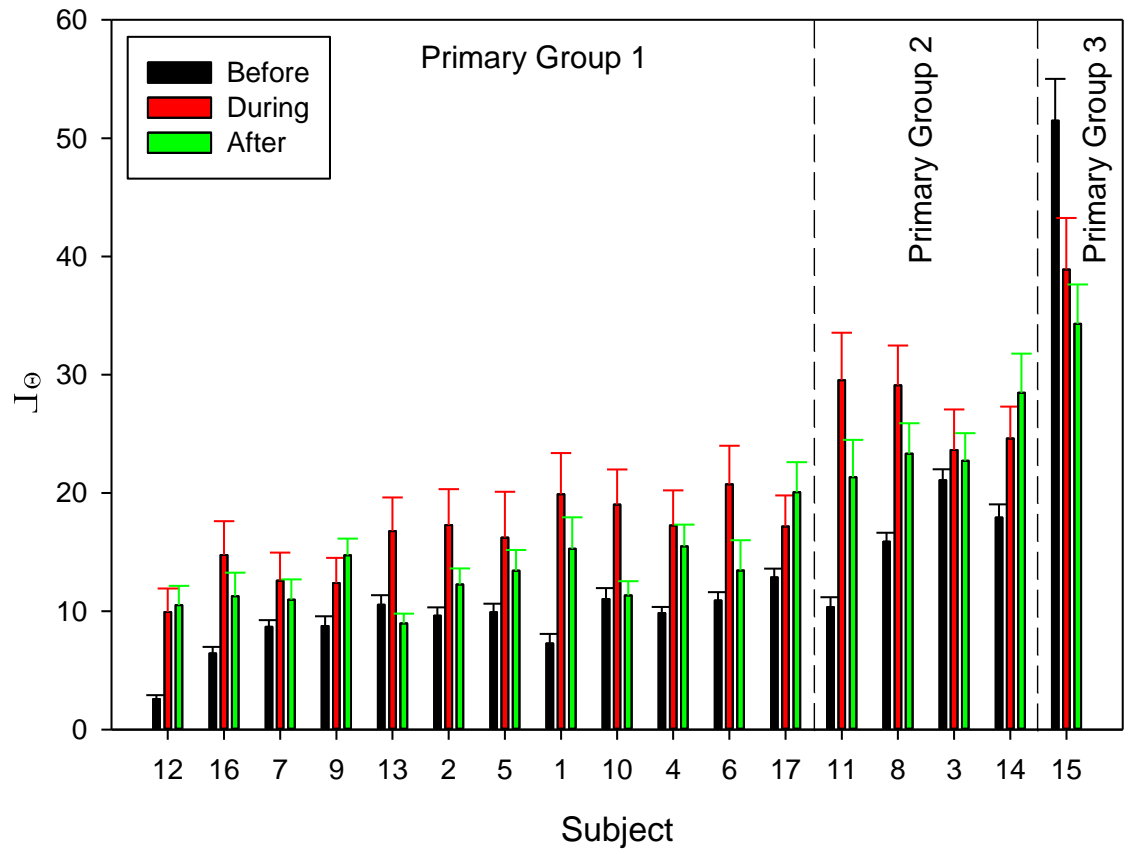

Figure 5. $\Gamma_{\Theta}$ by Subject and Segment

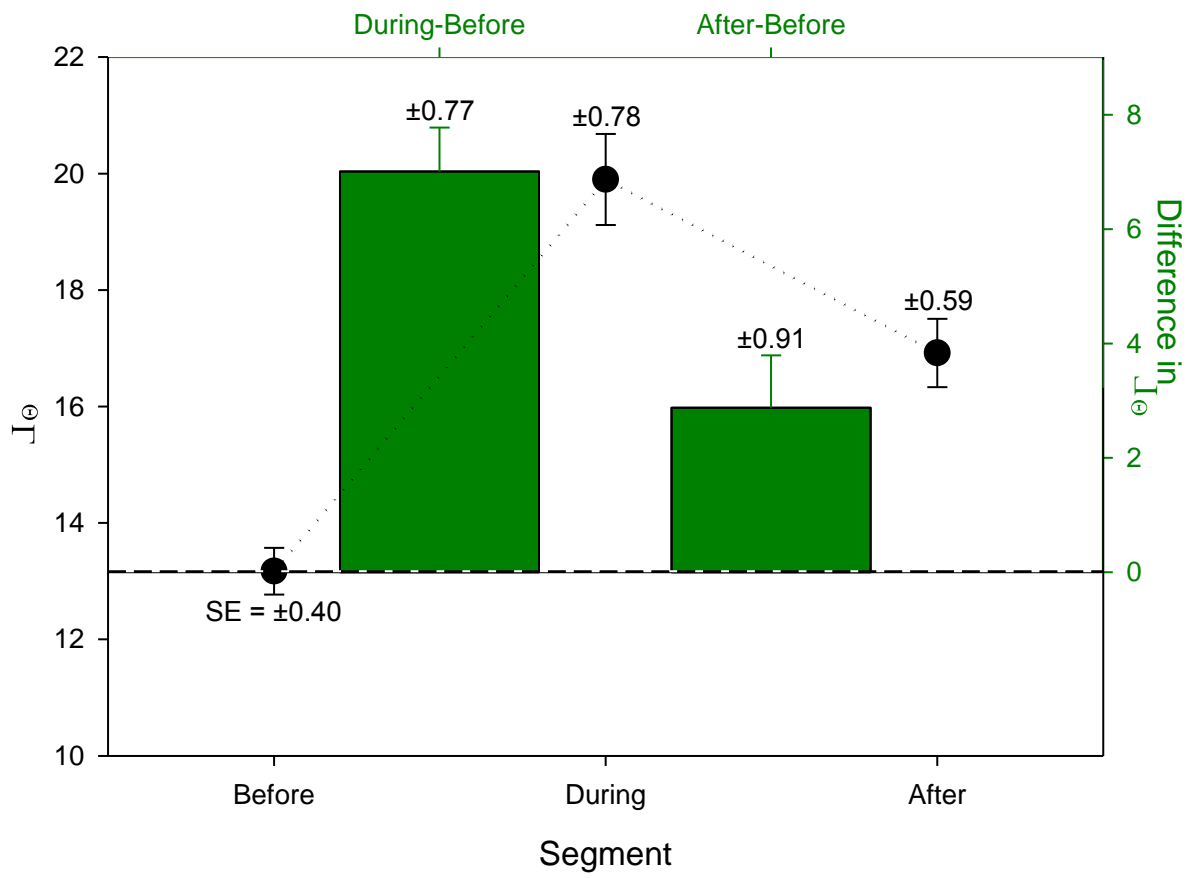

Figure 6. $\Gamma_{\Theta}$ by Segment

6

American Institute of Aeronautics and Astronautics 


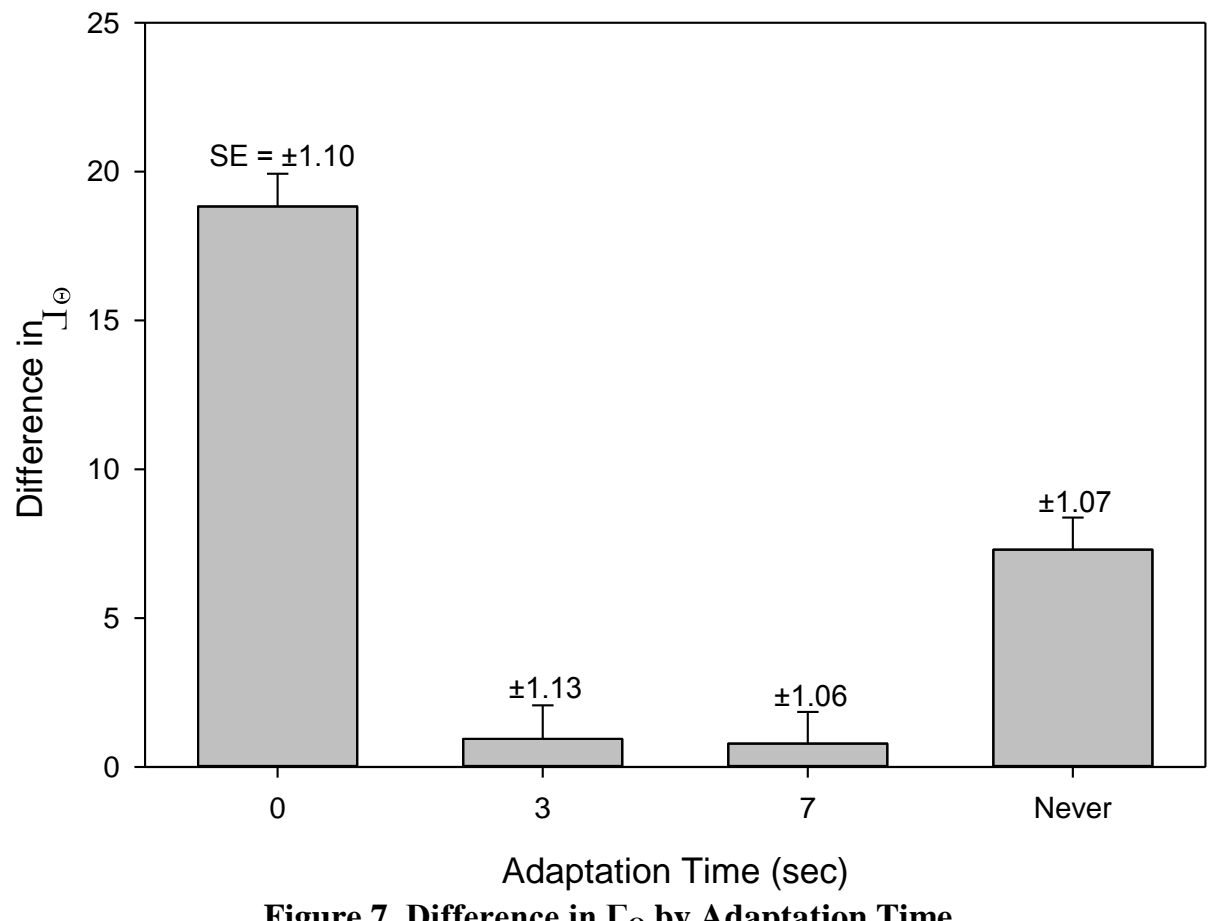

Figure 7. Difference in $\Gamma_{\Theta}$ by Adaptation Time

\section{Discussion and Future Research}

The simplest method, GDE, appears to have suitable accuracy in predicting pilot longitudinal stick inputs. This may allow for faster computation of its estimation gain parameter, $\Gamma_{\Theta}$, possibly approaching real-time computations. The methodology was also able to detect changes in pilot behavior with changing vehicle dynamics during a data run. However, this parameter was somewhat dependent on the pilot suggesting that the initial estimation gain may need to be calculated for each pilot before flying. This dependency, though, may be categorical based on general piloting methods of each pilot. This brings up the additional possibility that if the estimation gain parameter changes such that the difference suggests the pilot is now controlling the vehicle using a different method, then this may further strengthen the case that there is a problem someplace in the system. In general, the estimation gain parameter increased during the failure and then decreased after the failure. If given enough time, this decrease may approach the estimation gain to before-failure values. Therefore, this methodology should be able to detect changes in pilot behavior possibly in near real time. As part of current and future research, several learning methods are being applied to the existing data sets from LTE and ACE-PE to develop multiple models running in parallel to (i) determine if a particular learning methodology is better suited to short term pilot behavior predictions and (ii) if multiple methods are suitable, then they can be used to minimize false positives and provide better insight into causes of behavioral changes. If this line proves fruitful, then GDE can be used as a first alert since it can give almost real-time indication of behavioral changes. Even without potential contribution from learning-based models, the current GDE predictor can be used as part of noninvasive suite of measures detecting various stages of pilot incapacitation.

With other additional information from on-board vehicle state sensors, eye tracking sensors and possibly physiological measures ${ }^{30}$, it should be possible to determine if detected changes in piloting are due to a vehicle failure or issues with the pilot. If it is a piloting issue, such as excessive workload or inattention, less invasive remedial measures could be taken depending on the issue. For instance, if workload is high, the automation could direct the pilot's attention back to the aviating task while transferring some of the other tasks to the automation such as automatic completion of checklists ${ }^{31}$.

However, if the problem persists, such as a major fault/failure or the pilot is incapacitated, more invasive methods to save the aircraft may be needed such as an autonomous landing system which recognizes suitable airfields, calculates a safe trajectory to a particular airfield, notifies air traffic control while flying the new safe trajectory, and finally lands the aircraft. Determining when the automation should take over needs to be carefully considered. Obviously, if the pilot is incapacitated, determined via additional physiological measures and lack of response, then the automation taking over is justified. However, if the pilot is not fully incapacitated, then transferring control to automation becomes a more complex question. While the pilot may not be fully incapacitated her ability to competently 
fly may be highly impaired; however, several sources of corroborating evidence would be required. One such piece of evidence could be a large abrupt change in the estimation gain parameter or continuous change (outside a preset deadzone) in this parameter suggesting that the pilot is trying to adapt to a discrete event or degrading conditions. This information, in addition to physiological measures and changes in aircraft state information, may provide the evidence requiring the automation to take control of the aircraft from the pilot and conversely if it is appropriate to release control back to the pilot ${ }^{32-34}$.

Lastly, the analytical method described above, if similarly applied to autopilots, may also be able to detect software anomalies with the automation. The method applied would be similar as the one described for pilots - a sudden change or continuous changes in the estimation gain parameter but with no detected aircraft state anomalies. In this case, though, the automation might determine that it is best to hand full control to the pilot.

Therefore, developing an analytical model of the pilot that can be computed in near real time opens up the possibility of detecting and quantifying changes in piloting behavior. Combining this information with physiological data and aircraft state could further refine the function allocation between the pilot and the automation and could increase the possibility of safely determining when the autonomy should fully take over from an incapacitated pilot.

\section{Acknowledgments}

The authors would like to thank Lucas Hempley of Northrop Grumman for his help in programming the simulation and the experiment. The authors would also like to thank John Foster and Kevin Cunningham of NASA Langley Research Center for their help in tuning the aircraft simulation model for the two experiment described above. For insight into the estimation methods, the authors would like to thank Dr. Eugene Lavretsky, Senior Technical Fellow at Boeing.

This research was funded by the NASA's Aviation Safety Program - Vehicle Systems Safety Technologies project and NASA Langley Research Center's Autonomy Incubator.

\section{References}

${ }^{1}$ Bailey, R. E., and Mook, D. J. "Pilot Control Identification using Minimum Model Error Identification, AIAA 92-1121." 1992.

${ }^{2}$ Davidson, J. D., and Schmidt, D. K. "Modified Optimal Control Pilot Model for Computer-Aided Design and Analysis." Vol. NASA-TM-4384, NASA, 1992.

${ }^{3}$ Doman, D. B., and Anderson, M. R. "A Fixed-Order Optimal Control Model of Human Operator Response," Automatica Vol. 36, No. 3, 2000, pp. 409-418.

${ }^{4}$ Hess, R. A. "Obtaining Multi-Loop Pursuit-Control Pilot Models From Computer Simulation, AIAA-2007-247." 2007.

${ }^{5}$ Hess, R. A., and Mnich, M. A. "Identification of Pilot Dynamics from In-Flight Tracking Data, AIAA Paper 85-1945," AIAA Guidance, Navigation and Control Conference 1985. AIAA, Snowmass, CO, 1985, pp. 554-563.

${ }^{6}$ McRuer, D. T., and Jex, H. R. "A Review of Quasi-Linear Pilot Models," IEEE Transactions on Human Factors in Electronics Vol. HFE8, No. 3, 1967, pp. 231-249.

${ }^{7}$ McRuer, D. T., and Krendel, E. S. "Mathematical models of human pilot behavior." 1974.

${ }^{8}$ Kleinman, D. L., Baron, S., and Levison, W. H. "An Optimal Control Model of Human Response|Part 1: Theory and Validation," Automatica Vol. 6, No. 3, 1970, pp. 357-369.

${ }^{9}$ Hess, R. A. "Rudder Control Strategies and Force/Feel System Designs in Transport Aircraft," Journal of Guidance, Control, and Dynamics Vol. 28, No. 6, 2005, p. 12.

${ }^{10}$ Hess, R. A. "Unified Theory for Aircraft Handling Qualities and Adverse Aircraft-Pilot Coupling," J. Guidance, Control, and Dynamics Vol. 20, 1997, pp. 1141-1148.

${ }^{11}$ Schmidt, D. K. "Optimal Flight Control Synthesis Via Pilot Modeling," J. Guidance \& Control Vol. 2, No. 4, 1979, pp. 308312.

${ }^{12}$ Hess, R. A. "Simplified Approach for Modeling Pilot Pursuit Control Behavior in Multi-Loop Flight Control Tasks," Journal of Aerospace Engineering Vol. 220, No. G2, 2006, pp. 85-102.

${ }^{13}$ Hess, R. A. "A Preliminary Study of Human Pilot Dynamics in the Control of Time-Varying Systems," AIAA Modeling and Simulation Technologies Conference. Vol. AIAA-2011-6554, Portland, OR, 2011.

${ }^{14}$ Brieger, O., Ossmann, D., and Rüdinger, M. "A New Flight Test Technique for Pilot Model Identification," AIAA Atmospheric Flight Mechanics Conference and Exhibit. Vol. AIAA-2008-6556, Honolulu, HI, 2008, p. 16.

${ }^{15}$ Lampton, A., and Klyde, D. "Power Frequency - A New Metric for Analyzing Pilot-in-the-Loop Flying Tasks," Atmospheric Flight Mechanics Conference. Vol. AIAA-2011-6539, AIAA, Portland, OR, 2011, p. 21.

${ }^{16}$ Thompson, P., Bachelder, E., Klyde, D., Harris, C., and Brenner, M. "Wavelet-Based techniques for Improved Online Systems Identification," USAF Developmental Test and Evaluation Summit. Vol. AIAA-2004-6838, AIAA, Woodland Hills, CA, 2004.

${ }^{17}$ Thompson, P., Klyde, D., and Brenner, M. "Wavelet-Based Time-Varying Human Operator Models," Atmospheric Flight Mechanics Conference and Exhibit. Vol. AIAA-2001-4009, AIAA, Montreal, Canada, 2001, p. 12. 
${ }^{18}$ Zaal, P., Pool, D. M., Mulder, M., and van Paassen, M. M. "Multimodal Pilot Control Behavior in Combined Target-Following Distrubance-Rejection Tasks," AIAA Modeling and Simulation Technologies Conference. Vol. AIAA-2009-6057, Chicago, IL, 2009, p. 18.

${ }^{19}$ Zaal, P., Pool, D. M., Mulder, M., and van Paassen, M. M. "Multimodal Pilot Model Identification in Real Flight," AIAA Modeling and Simulation Technologies Conference. Vol. AIAA-2009-6028, AIAA, Chicago, IL, 2009, p. 20.

${ }^{20}$ Zaal, P., and Sweet, B. "Estimation of Time-Varying Pilot Model Parameters," AIAA Modeling and Simulation Technologies Conference. Vol. AIAA-2011-6474, AIAA, Portland, OR, 2011.

${ }^{21}$ Gore, B. F. "Man-Machine Integration Design and Analysis System (MIDAS) v5: Augmentations, Motivations, and Directions for Aeronautics Applications," 1st International Workshop on Human Modelling in Assisted Transportation. Belgirate, Italy, 2011, p. 11.

${ }^{22}$ Gore, B. F., and Jarvis, P. A. "New Integrated Modelling Capabilities: MIDAS' Recent Behavioural Enhancements," SAE 2005 Transactions Journal of Passenger Cars: Electronic and Electrical Systems Vol. SAE 2005-01-2701, 2005, p. 8.

${ }^{23}$ Shively, R. J. "Re-Design and Beat Testing of the Man-Machine Integration Design and Analysis System: MIDAS," Digital Human Modeling for Design and Engineering Conference and Exposition. Hague, Netherlands, 1999.

${ }^{24}$ Trujillo, A. C., and Gregory, I. M. "Preliminary Exploration of Adaptive State Predictor Based Human Operator Modeling," 2012 AIAA Modeling and Simulation Technologies Conference. AIAA, Minneapolis, MN, 2012.

${ }^{25}$ Trujillo, A. C., and Gregory, I. M. "Adaptive Controller Adaptation Time and Available Control Authority Affects on Piloting," 17th International Symposium on Aviation Psychology. Dayton, OH, 2013.

${ }^{26}$ Trujillo, A. C., and Gregory, I. M. "Pilot Preferences on Displayed Aircraft Control Variables," HCI International 2013. Las Vegas, NV, 2013.

${ }^{27}$ Trujillo, A., Gregory, I. M., and Hempley, L. "Adaptive Controller Effects on Pilot Behavior," To be presented at the IEEE 2014 International Conference on Systems, Man, and Cybernetics. IEEE, San Diego, CA, 2014.

${ }^{28}$ Lavretsky, E. "System Identification (ID): CDS 270-I." Caltech, Caltech, 2008, p. 93.

${ }^{29}$ IBM. "IBM SPSS Statistics Version 22." 22.0.0.0 ed. Vol. 64-bit, IBM, 2013.

${ }^{30}$ Brackens, B. "Electronic Band Aid to Measure Body Performance Through Sweat," Air Force Print News Today, 2014.

${ }^{31}$ Comstock, J. R., and Arnegard, R. J. "The Multi-Attribute Task Battery for Human Operator Workload and Strategic Behavior Research." NASA, NASA Langley Research Center, 1992, p. 99.

${ }^{32}$ Williams, D. M., Lohr, G. W., and Trujillo, A. C. "Wake Turbulence Mitigation for Arrivals (WTMA)," 26th Congress of International Council of the Aeronautical Sciences. Anchorage, AK, 2008.

${ }^{33}$ Swihart, D. E., and Griffin, E. "Automatic Ground Collision Avoidance System (AUTO GCAS)," 13th WSEAS International Conference on SYSTEMS. World Scientific and Engineering Academy and Society, 2009, p. 5.

${ }^{34}$ Swihart, D. E., and Skoog, M. A. "Automatic Collision Avoidance Technology (ACAT)," UVS 2007. Blyenburgh \& Co., Paris, France, 2007, p. 24. 